\title{
Fistulae of the Canal Lateral Due to Cholasteatomatous Otitis About 5
} Cases

\author{
Bamine Hamed*, Gharnati Karim, Ridal Mohamed, Alami MN
}

Département of ENT, UHC Hassan II Fes Marroco

DOI: $\underline{10.36347 / \text { sasjs.2021.v07i05.001 }}$

| Received: 16.03.2021 | Accepted: 21.04.2021 | Published: 04.05.2021

*Corresponding author: Bamine Hamed

Abstract

Our study is retrospective on 5 cases of fistulae of the lateral semi-circular duct (CSCL) complicating chronic cholesteatomatous otitis media, operated in the otolaryngology department of the CHU HASSAN II of Fez, over a period of 4 years from January 2014 to December 2017. The age of our patients ranged from 6 years to 65 years with a mean of 26 years, and a clear male predominance. The consultation period ranged from 1 year to 15 years. Othorrhea and vertigo were consistent in all our patients, combined with mixed deafness in 3 patients. The otoscopy revealed several aspects; going from image Typical a cholesteatoma of the middle ear with epidermal scales in the middle ear emerging from a perforation or a tympanic retraction pocket, or an attical cholesteatoma on a stage III retraction pocket. Ossicular lysis was evident in 3 of patients. Peripheral vestibular syndrome was noted in all of our patients. Neurological examination found a case of facial paralysis. Concerning the functional impairment; 2 of our patients had pure transmissive deafness, the remaining 03 patients had mixed deafness. Diagnosis labyrinthine fistulae has been clinically evoked and surgically confirmed. All our patients received preoperative CT scan. MRI has a complementary and fundamental role in the diagnosis of some internal ear disorders, it was requested in one patient. Treatment of fistulae (CSCL) was achieved by plugging the breach with temporal aponevrosis associated with the treatment of Chronic cholesteatomatous otitis. In our series, all our patients received general antibiotic therapy adapted to the antibiogram, 3 patients received treatment with open tympanoplasty wall down patients received tympanoplasty by closed technique. The surgical follow-ups were simple.

Keywords: Cholesteatoma, Fistula, Labyrinthine, Surgery, Deafness.

Copyright $\odot 2021$ The Author(s): This is an open-access article distributed under the terms of the Creative Commons Attribution 4.0 International License (CC BY-NC 4.0) which permits unrestricted use, distribution, and reproduction in any medium for non-commercial use provided the original author and source are credited.

\section{INTRODUCTION}

From a nosological point of view, we distinguish two entities; labyrinthine bone lyses and perilymphatic fistulae. In the first case, the destruction only affects the bony labyrinth, without affecting the endosteum. In the second, the labyrinthine fistula bare or even reaches the membranous labyrinth [2]. Lateral circular is the most common location. Fistulae of the (CSCL) Complicates 5 to $10 \%$ of otitis median Chronic cholesteatomatous disease $[1,2]$.

\section{MATERIAL AND METHOD}

This is a retrospective study involving 05 patients with a fistula of the (CSCL) complicating choletheatomatous otitis, collected in the ORL department of the CHU Hassan II in Fez, Morocco, between January 2014 and December 2017. The statistical analysis was carried out using the SPSS software.

\section{RESULT \\ Epidemiological and clinical aspects Observation $\mathrm{N}^{\circ} 1$}

5 year old child without special ATCD, admitted for care peripheral vestibular syndrome appeared a month ago. The left ear otoscopy objective the presence of a retro tympanic material of whitish colour healthy tympanic membrane, normal right ear, evoking a congenital choletheatom. TDM: full body with left CSCL lysis.

\section{Observation $\mathbf{N}^{\circ} 2$}

Patient of 9 years followed for chronic bilateral otitis media. Objective otoscopy the presence of purulent otorrhea with epidermal debris in the left ear and lysis of the descending branch of the hammer. On the right, simple tympanic perforation. TDM: a demineralized aspect of the tegmen tympani that is lysed in places with lysis of the left CSCL 


\section{Observation $\mathbf{N}^{\circ} \mathbf{3}$}

Patient of 49 years followed for right chronic otitis media for 10 years aggravated 2 months ago by facial paralysis then two weeks ago by peripheral vestibular syndrome.

Otoscopy shows the presence of a posterior superior perforation with spontaneous Atticotomy and extensive ossicular lysis. TDM: lysis of the back wall of the rock and the right CSCL. It also shows a lysis of the bony shell of the facial canal at the level of its 2 nd and 3rd portion.

\section{Observation $\mathrm{N}^{\circ} \mathbf{4}$}

Patient 65 years followed for left chronic otitis media with otoscopy; presence of an Atticale retraction pocket leaving behind pus mixed with scales. Sign of positive fistula TDM: filling of the cash register tympanum with lysis of mastoid cells and left CSCL.

\section{Observation $\mathrm{N}^{\circ} 5$}

21-year-old patient followed for otitis media chronic right aggravated 2 weeks ago by peripheral vestibular syndrome with signs of positive fistula. The otoscopy shows the presence of a polyp the external ear canal preventing the visualization of the right eardrum. CT: Atticale filler with lysis of the CSCL and suspicion of membranous damage confirmed by MRI

Our series consists of 04 men and one woman. With an average age of 26 years, the extreme ages are 05 years and 65 years. 4 of patients are from a low socioeconomic background. The consultation time in our patients varies from 1 to 15 years, with an average consultation time of 4 years, making diagnosis often late. It is noted that otorrhea is by far the most frequently observed reason for consultation; At the time of diagnosis, 04 of our patients have otorrhea, vertigo in 4 patients, hypoacousia in 3 patients, facial paralysis one case. The side most often hit was the left side. The otoscopy revealed several aspects; going from image Typical a cholesteroma of the middle ear with epidermal scales in the middle ear emerging from a perforation or a tympanic retraction pocket, or an attical cholesteroma on a stage III retraction pocket. Ossicular lysis was evident in 03 patients. Neurological examination found facial paralysis in one case. Concerning the functional impairment; 02 of our patients had pure transmissive deafness, 3 patients had mixed deafness. The CT shows hypo dense fillings at the convex edges of the eardrum case in 4 patients and a lysis of the CSCL in all of our patients.

The MRI was performed on a patient for suspicion of membranous damage on the CT; she objectified a membranous labyrinthine fistula of the CSCL.

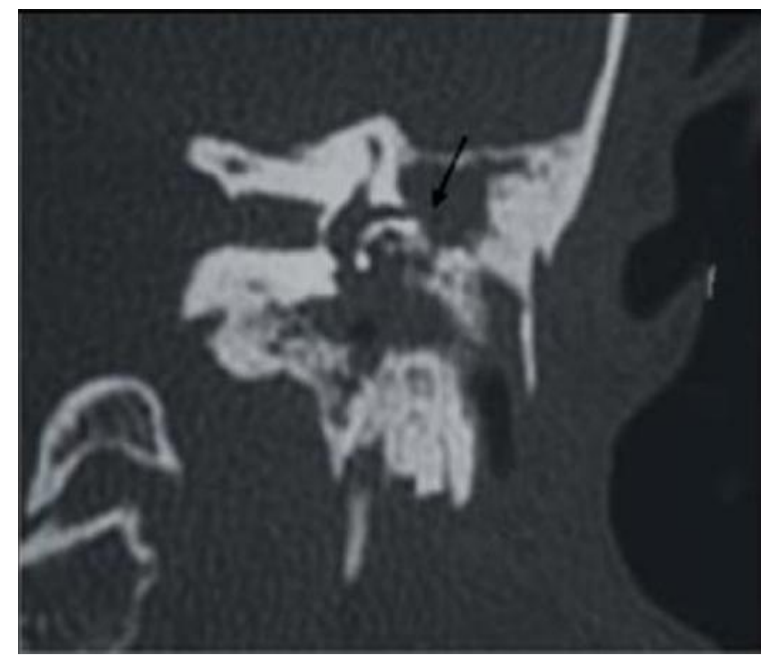

Fig-1: Image showing a lysis of the semi-channel -lateral circular, with lysis of the upper wall of the temporal bone

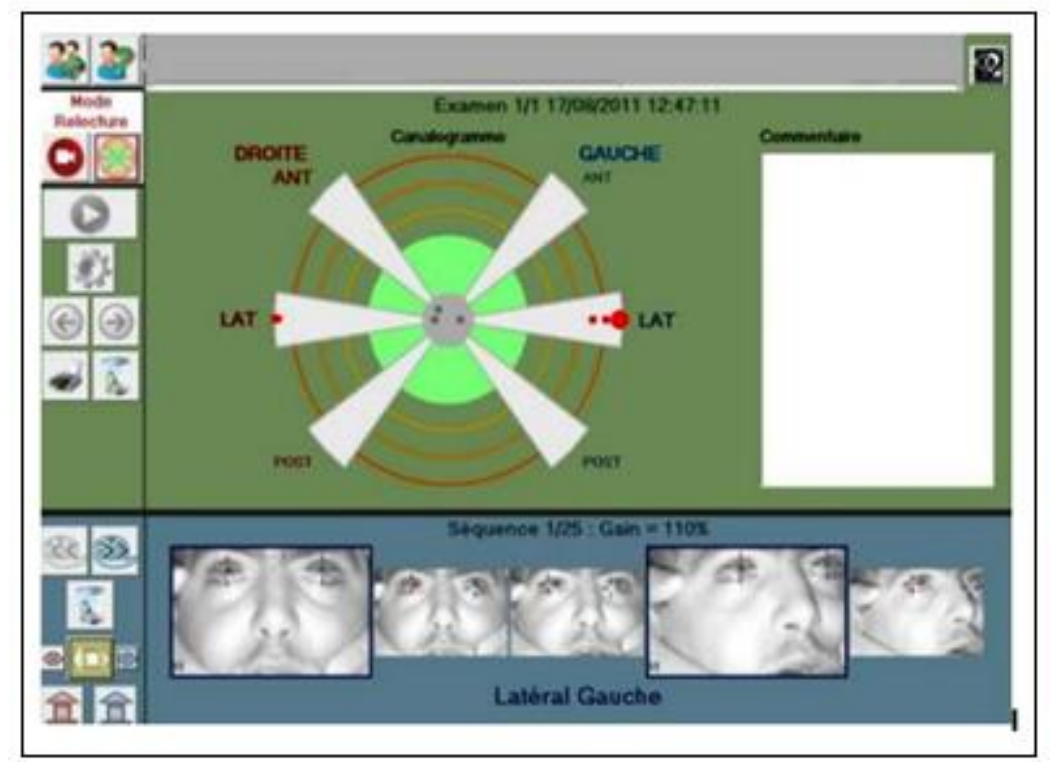

Fig-2: déficit monocanalaire latérale bilatéral sur les hautes fréquences 


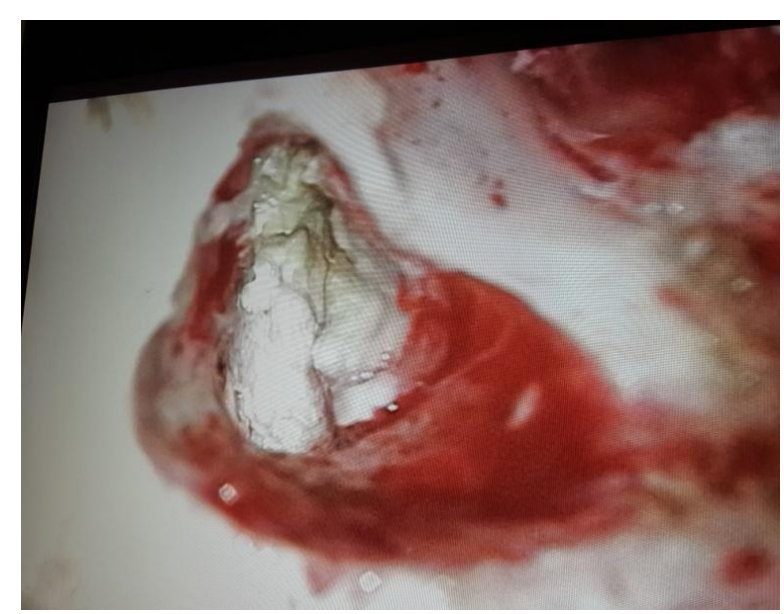

Fig-3: intraoperative image d' a cholesteatoma of the posterior cavities

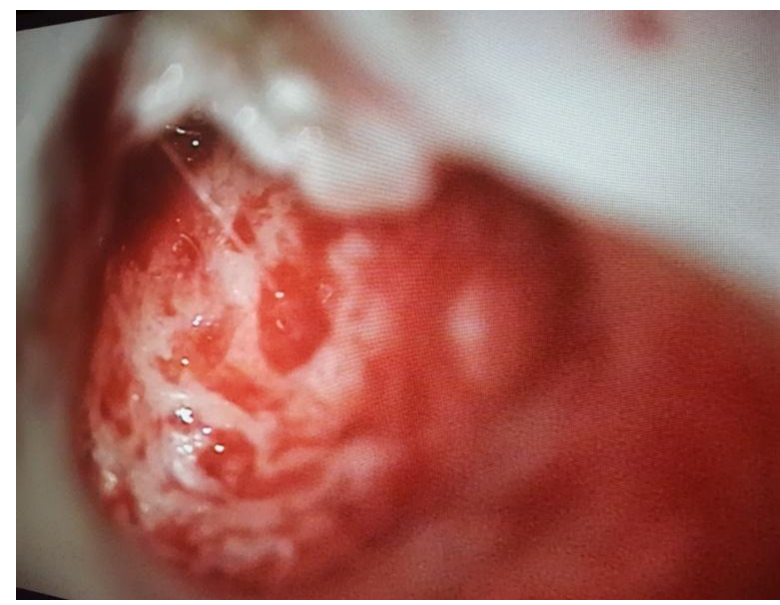

Fig-4: intraoperative image showing a CSCL fistula

\section{Therapeutic Aspect \\ Medical Treatment}

All patients received general antibiotic therapy with amoxicillin+clavulanic acid $(80 \mathrm{mg} / \mathrm{kg} /$ day in 3 doses in children, $2 \mathrm{~g} /$ day in 2 doses in adults), or fluoroquinolone (>14 years, ciprofloxacilin $1 \mathrm{~g} /$ day in 2 doses) and local antibiotic therapy with fluoroquinolone in ear drops for 10 days combined with otological aspiration. The antibiotic therapy was subsequently adjusted according to the antibiogram. Corticosteroid therapy at a dose of 1 to $1.5 \mathrm{mg} /$ day was indicated in patients with facial paralysis for about 10 days.

\section{Surgical Treatment}

In our series, 03 patients had been treated with open surgery while 02 patients were treated with closed surgery. The fistula was filled by temporal aponeurosis in 4 cases and perichondria in 1 case. In peroperatory, the lysis of the semi-canal-lateral circular was reported in all cases, dehiscence of the tegmen tympani with proincidence of the meninges was noted in 03 cases, lysis of the facial canal in peroperatory one case. Exploration of the ossicular chain in peroperatory showed Ossicular lysis in 04 patients. Functional gesture was delayed due to an extension of the cholesteratoma to the oval niche or an unfavourable condition of the mucosa. No decompression of the facial nerve was performed, treatment of facial paralysis was based on removal of the causal cholesteatom and corticosteroid therapy

\section{Follow-Up}

In the post-operative period, all our patients benefited from an antibiotic therapy based on protected amoxicillin and ear drops, early discharge was recommended, the average length of hospitalization was 5 days. Post-operative follow-ups were mostly simple; Only 1 case of wound infection was noted, which was managed by antibiotic therapy and local care. Postoperative mortality was zero.

\section{DISCUSSION}

The majority of studies have shown that the attainment of isolated lateral AUC ranged from $57 \%$ to $91 \%[1,2,6]$. The tendency of the cholesteratoma to develop in the antro cavities -atticales explains this topographical preponderance. However, other locations have been observed. They may be isolated or associated with lateral CSC damage. They are found in the literature with varying frequencies depending on the series: the promontory in $6.9 \%$ [7], the oval window in $7.7 \%$ [8], or the upper semi-circular canal in 5.4\% [9]. For some, the site of the fistula would have a prognostic value for postoperative hearing.

Thus, a cochlear or vestibular fistula would have a worse prognosis than a damage to the semicanals -circulars. Similarly, the risk of hearing loss would be greater if the upper or posterior semicircular canal were affected compared to the lateral semicircular canal [4]. Preoperative diagnosis is complex and controversial. There is no specific test for the diagnosis of LF. The fistula sign or Hennebert's sign, if it is relatively specific, is in contrast not very sensitive with a false-negative rate exceeding 50\%. This sign is found in the literature in 22 to $53 \%$ of cases $[2,12]$. In our series this sign was present in $48 \%$ of cases. According to the literature, the clinical picture is made up of a dragging otorrhea associated with a progressive worsening deafnessThese two signs point more towards the diagnosis of chronic cholesteatomatous otitis media and are not specific to labyrinthine fistulas [2, 10]. Moreover, the vertigo reported during the interrogation by half of the patients is not the major reason for consultation in most cases. labyrinthine fistula since many authors reported normal bone curves in their series $[3,4]$. Computed tomography (CT) is currently the reference imaging of cholesteatoma and allows the diagnosis of complications related to bone erosion. Technical progress (helicoidal acquisition) and the emergence of forensic requirements are now encouraging the systematic implementation of a fine-cut tomography in the pre-operative assessmentIts sensitivity to the positive diagnosis of labyrinthine fistula ranged from $90 \%$ to $100 \%$ in the literature [13, 
14]. According to the classification of Dornhoffer and Milewski:

- Type I corresponds to a destruction of the periosteum and the medial layer of the bone wall.

- Type II is characterized by the destruction of the inner layer of the bony labyrinth making a direct contact of the cholesteatom with the perilymphatic space and the membranous labyrinth.

- Type III corresponds to a destruction of all the layers of the bone labyrinth with invasion of the cholesteratoma matrix into the endolymphatic space.

In our series, fistula was type I (45\%), 18 type II (43\%) and 5 type III (12\%). MRI would be the most reliable radiological examination for the diagnosis of perilymphatic fistula [13]. The choice of surgical technique and whether or not the complete removal of the matrix has been the subject of much debate in the past. cochleated and thus protect the auditory function Others advocated complete removal of the matrix because of the potential for osteolysis of the cholesteroma and to avoid further complications [12]. The authors recommend safe removal of the matrix of a small fistula (less than $2 \mathrm{~mm}$ in diameter), while the matrix of a fistula larger than $2 \mathrm{~mm}$ in diameter should be left in place. These same authors have stipulated that the decision depends on the location and size of the fistula, the state of the auditory function, but also on the surgeon's experience [12]. In a literature review of 1018 patients with labyrinthine fistula secondary to cholesteratoma of the middle ear [15] showed that auditory results were comparable between those who had a complete removal of the matrix and those whose removal was incomplete. In other words, the two surgical procedures would not appear to have an impact on auditory function. Today, the Consensual attitude consists in practicing, whenever possible, the removal of the matrix $[8,12]$ having previously informed the patient of the auditory risk associated with the gesture. In addition, the removal of the matrix had to be performed at the end of the procedure, in order to leave the labyrinth bare for a minimum period of time [2]. This approach is the surgical attitude adopted in our department. The choice between open or closed technique is also controversial, as the lowering of the wall is independent of whether or not there is a labyrinthine fistula It is more dependent on the extent of the cholesteratomatous lesions. In addition, the choice of technique does not affect the quality of the postoperative bone curve. In these circumstances, the use of a closed technique should be recommended except in special circumstances (multi-ear -operated, multiple fistulae, single ear) or when it is considered to leave the matrix in place (large fistula of the posterior canal, promontorial, cochlear), in order to facilitate subsequent monitoring [2]. Although the reconstruction techniques differed among the authors, there is, however, a near consensus on the usefulness of bone powder collected at the surgical site covered by a temporal aponeurosis or periosteal tissue fragment [16]. Grewal et al., recommended the use of temporal aponeurosis instead of bone powder for large fistulae due to the risk of penetrating and spreading into the membranous labyrinth [5]. The risk of postoperative cophosis averages $10 \%$ and is often considered unpredictable. However, for some authors, the risk of hearing seems to be correlated with the size but also with the location of the fistula. Indeed, the auditory prognosis is more reserved for cochlear seat fistulae. For this location, a certain caution is required regarding the removal of the cholesteratomatous matrix, against the fistulae of the lateral SCC, which are considered to have a good prognosis [17].

\section{CONCLUSION}

CSCL fistula often presents as a complication of cholesteatoma. She's clinically suspect and confirmed on the CT and peroperative. Rapid diagnosis and early management improve the prognosis.

Declaration of Relationships of Interest: The authors state that they do not have any ties of interest.

Contributions by the authors: All authors contributed to the conduct of this work. All authors also state that they have read and approved the final version of the manuscript.

\section{REFERENCES}

1. Durko M, Durko T. Labyrinthine fistulas due to the chronic cholesteatomatous otitis mediacontroversial issues on diagnosis and surgical treatment. Int Congr Ser. 2003.

2. Portier F, Lescanne E, Racy E, Nowak C, Lamblin B, Bobin S. Prise en Charge des Fistules Labyrinthiques Cholestéatomateuses: A Propos de 22 Cas. J Otolaryngol. 2005.

3. Manolidis S. Complications associated with labyrinthine fistula in surgery for chronic otitis media. Otolaryngol Neck Surg. 2000.

4. Faramarzi AH, Heydari ST, Rusta M. The prevalence of labyrinthine fistula in chronic otitis media surgery in Shiraz, Southern Iran. Iran Red Crescent Med J. 2011.

5. Grewal DS, Hathiram BT, Dwivedi A, Kumar L, Sheth K, Srivastava S. Labyrinthine fistula: a complication of chronic suppurative otitis media. J Laryngol Otol. 2003.

6. Gomaa MA, Abdel Karim ARA, Abdel Ghany HS, Elhiny AA, Sadek AA. Evaluation of temporal bone cholesteatoma and the correlation between high resolution computed tomography and surgical finding. Clin Med Insights Ear Nose Throat. 2013.

7. Martin C, Martin H, Prades JM, Perron X, Bertholon P. Cholesteatoma and labyrinthine fistula. Rev Laryngol - Otol - Rhinol. 1989.

8. Pulec JL. Labyrinthine fistula from cholesteatoma: surgical management. Ear Nose Throat J. 1996. 
9. Magliulo G, Terranova G, Varacalli S, Sepe C. Labyrinthine fistula as a complication of cholesteatoma. Am J Otol. 1997.

10. Dornhoffer JL, Milewski C. Management of the open labyrinth. Otolaryngol Head Neck Surg. 1995; 112:410-4.

11. Sanna M, Zini C, Bacciu S, Scandellari R, Delogu P, Jemmi G. Management of the Labyrinthine Fistula in Cholesteatoma Surgery. ORL. 1984.

12. Moriyama H, Honda Y, Huang CC, et al. Bone resorption in cholesteatoma: epithelialmesenchymal cell interaction and collagenase production. Laryngoscope 1987.

13. Gormley PK. Surgical management of labyrinthine fistula with cholesteatoma. J Laryngol Otol. 1986.

14. Ritter FN. Chronic suppurative otitis media and the pathologic labyrinthine fistula. Laryngoscope. 1970.

15. Debruyne F, Vantrappen G, Feenstra L, Hermans B, Baert A. Computed tomographic imaging of repaired fistulas of the lateral semicircular canal. The American journal of otology. $1994 \mathrm{Jul}$ $1 ; 15(4): 549-50$.

16. Ars B, Claes J, Casselman J, Ars-Piret N. Preservation of cochlear function after extensive labyrinthine destruction. The American journal of otology. 1996 Jan 1;17(1):40-5.

17. Gamoletti R, Sanna M, Zini C, Taibah AK, Pasanisi E, Vassalli L. Inner ear cholesteatoma and the preservation of cochlear function. The Journal of Laryngology \& Otology. 1990 Dec;104(12):9458.

18. Copeland BJ, Buchman CA. Management of labyrinthine fistulae in chronic ear surgery. Am J Otolaryngol. 2003.

19. Baylan MY, Yilmaz U, Akkus Z, Topçu I. Assessment of Bone Conduction Thresholds after Surgical Treatment in Patients with Labyrinthine Fistula. Turkish Archives of Otorhinolaryngology. 2018;56(2):89-94. 